\title{
Effectiveness of Cognitive-Behavioral Therapy on Executive Function and Experiential Avoidance in Veterans with Post-Traumatic Stress Disorder
}

\section{ART ICLE INF O}

\section{Article Type}

Original Research

\section{Authors}

Shafiei M.* MSc,

Sadeghi M. ${ }^{1} P h D$,

Ramezani $S^{2} M S c$

How to cite this article
Shafiei M, Sadeghi M, Ramezani
S. Effectiveness of Cognitive-Be-
havioral Therapy on Executive
Function and Experiential Avoi-
dance in Veterans with Post-Tr-
aumatic Stress Disorder. Iranian
Journal of War \& Public Health.
2017;9(4):169-175.

*Psychology Department, Literature \& Humanities Faculty, University of Lorestan, Khoramabad, Iran ${ }^{1}$ Psychology Department, Literature \& Humanities Faculty, University of Lorestan, Khoramabad, Iran ${ }^{2}$ Consultation and Rehabilitation Department, Education \& Psychology Faculty, Mohaghegh Ardebili University, Ardabil, Iran

\section{Correspondence}

Address: Psychology Department, Literature \& Humanities Faculty, University of Lorestan, Daneshgah Street, Khoramabad, Iran

Phone: +98 (66) 33120627

Fax: -

masumeh.shafiei62@gmail.com

\section{Article History}

Received: February 18, 2017

Accepted: May 23, 2017

ePublished: November 6, 2017

\section{A B S T RAC T}

Aims Impairment in executive functions can result in impaired self-regulatory behavior and social skills deficiency, and experiential avoidance is a strong predictor of psychological signs and symptoms. This study was aimed to evaluate the effectiveness of cognitive-behavioral therapy on executive function and experiential avoidance among veterans with post-traumatic stress disorder (PTSD).

Materials \& Methods This study was conducted in a quasi-experimental, pretest-posttest design with control group in 2017. The studied population was included all male veterans with post-traumatic stress disorder referred to Quds hospital in Sanandaj. 40 veterans were selected using available sampling method and were divided randomly in two experimental and control groups. The Wisconsin card sorting test (WCST) and acceptance and action questionnaire (AAQ) were used to collect information. The data were analyzed using SPSS 20 software by multivariate analysis of covariance (MANCOVA).

Findings After controlling the pre-test scores, the results showed that the mean scores of experimental group in number of categories and preservative error as the sub-components of executive function, and experiential avoidance were significantly lower than the control group $(\mathrm{p}<0.01)$.

Conclusion Cognitive-behavioral therapy is efficient to increase executive function and to reduce experiential avoidance among veterans with PTSD.

Keywords Cognitive-Behavioral Therapy; Executive Function; Experiential Avoidance; Veterans;
Stress Disorders, Post-Traumatic

\section{I T A T I O N L I N KS}

[1] Diagnostic and statistical manual ... [2] Predictive factors of chronic post-traumatic [3] Cognitive therapy for post-traumatic ... [4] A cognitive-behavioral group program [5] Psychological interventions for ... [6] Investigating symptoms of post traumatic ... [7 An in depth look into PTSD depression ... [8] Examining the broader psychosocial effects ... [9] Drinking motives and PTSD-related ... [10] The role of mindfulness as approachbased ... [11] PTSD, alcohol dependence, and conduct ... [12] An examination of sleep quality ... [13] PTSD symptoms and suicide ideation ... [14] Alexithymia and DSM-IV personality ... [15] Alexithmia in women and men ... [16] Aprospective study of alexithymia in ... [17] Relationship between anxiety sensitivity ... [18] Nature's role in predicting quality ... [19] Forster. Executive Functioning ... [20] Executive function in posttraumatic stress ... [21] Psychophysiological responses to an ... [22] Experiental avoidance and behavioral ... [23] The role of experiential avoidance ... [24] Peritraumatic dissociation and experiential ... [25] Experiential avoidance as a moderator ... [26] The comparison of experimental ... [27] Relationship of service members' deployment ... [28] The interactive effect of cognitive ... [29] Cognitive-behavioral intervention ... [30] The current state of cognitive ... [31] Mood disorders: Cognitive ... [32] Just showing up is not ... [33] The effects of cognitive behavioral ... [34] Religious vs. Conventional Cognitive Behavioral Therapy for Major ... [35] Physiological and behavioral indices of emotion dysregulation ... [36] Cognitive behavioral and pharmacological ... [37] Changes in the self during cognitive ... [38] Effectiveness of brief VR treatment for PTSD in ... [39] Measuring experiential avoidance: A preliminary ... [40] Executive functions and the obsessivecompulsive disorder ... [41] Cognitive function across manic or hypomanic, depressed ... [42] Preliminary psychometric properties ... [43] Timing matters: change ... [44] On the evolutionary origins ... [45] Play: Essential for all children. A position paper of the association for childhood education ... [46] Healing traumatized children ... 
اختلال استرس يس از سانحه مىشود و زندگى آنها را مختل

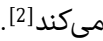

شايعترين سن ابتلا به اين اختلال اوايل بزركسالى است. مردان و وإئل

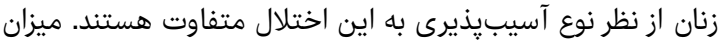

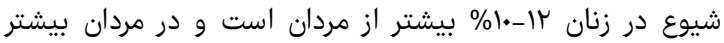

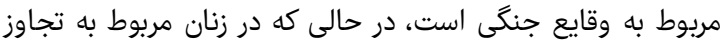

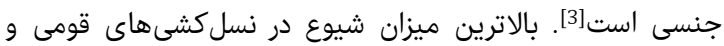

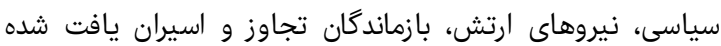

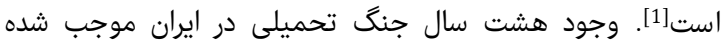

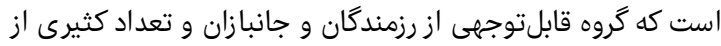

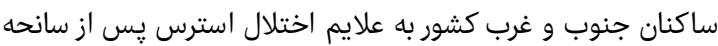
(PTSD)

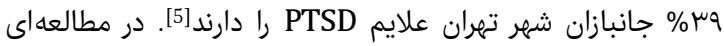

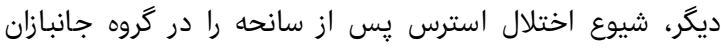

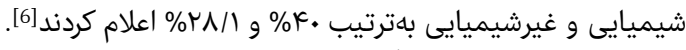

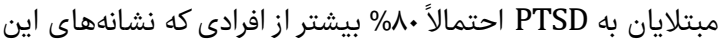

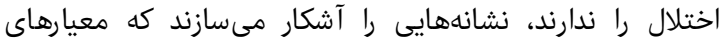

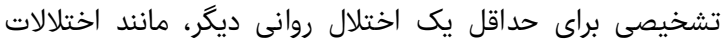

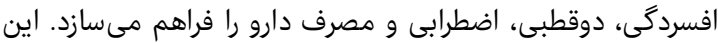

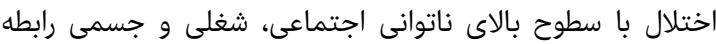

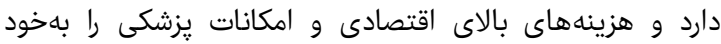

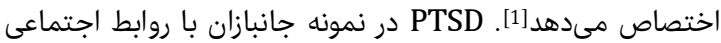

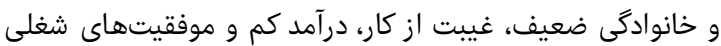

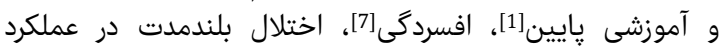

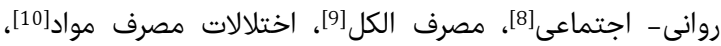

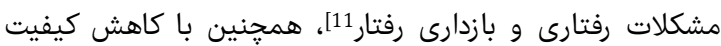

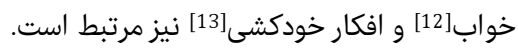

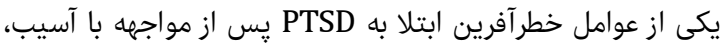

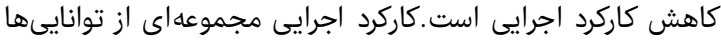

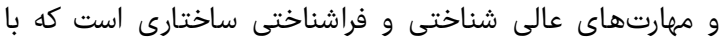

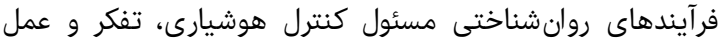

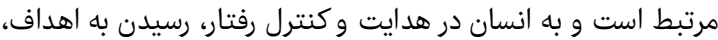

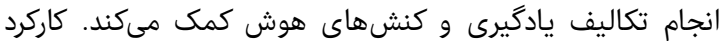

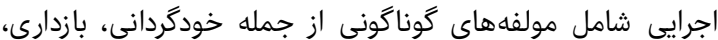

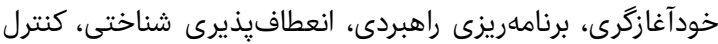

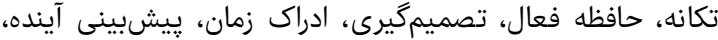

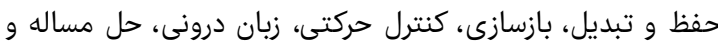

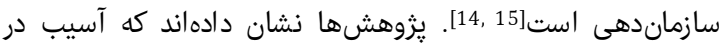

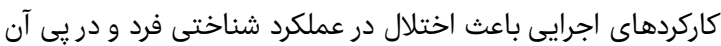

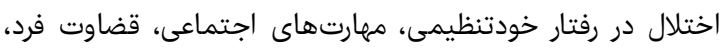

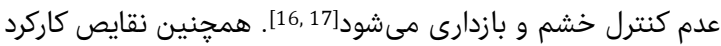

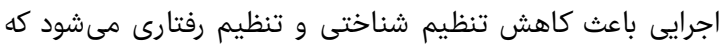

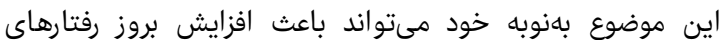

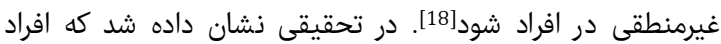

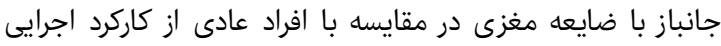

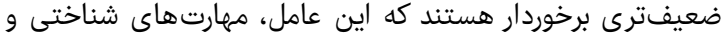

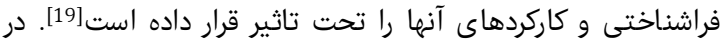

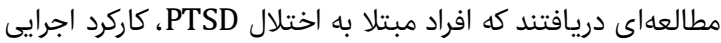

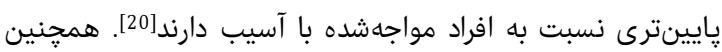

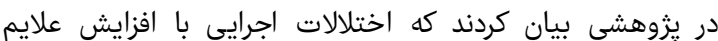

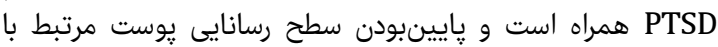

اثربخشى درمان اجرايى و اجتناب تجربى جاني دانبازان مبتلا به اختلال استرس يس از سانحه

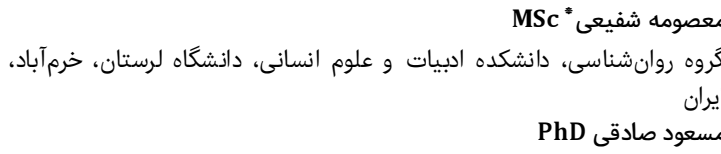

كروه روانشناسى، دانشكده ادبيات و علوم انسانى، دانشكاه لرستان، خرمآباد،

ايران

MSc Mكوفه رمضانى

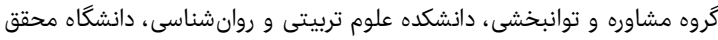

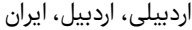

קكيده

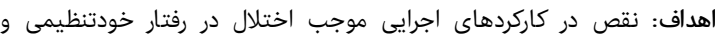

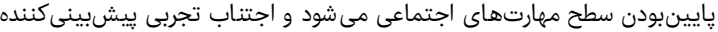

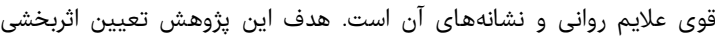

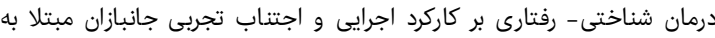

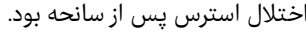

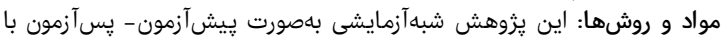

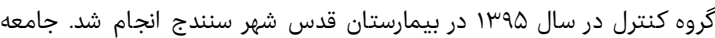

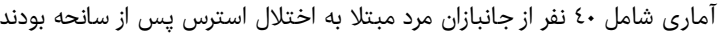

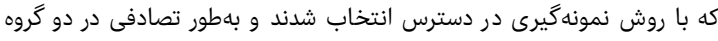

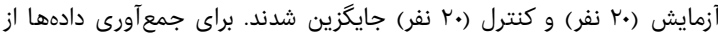

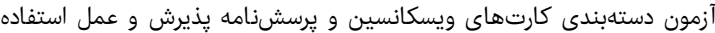

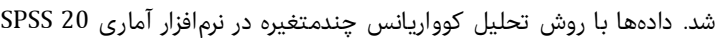

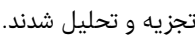

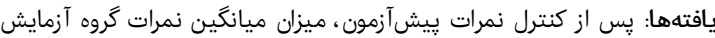

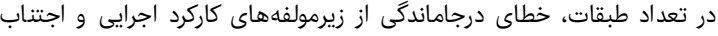

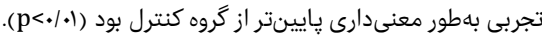

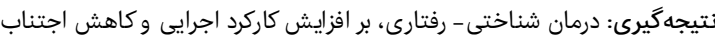

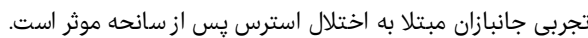

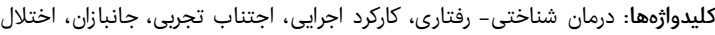

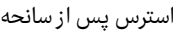

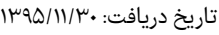

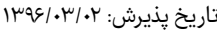

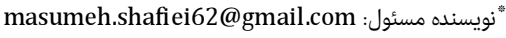

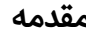

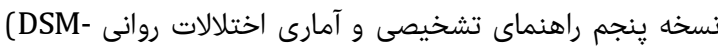

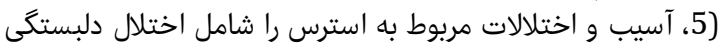

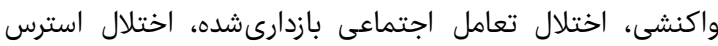

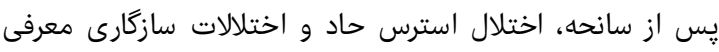

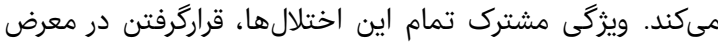

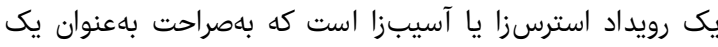

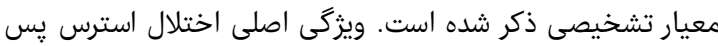

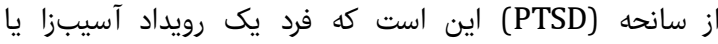

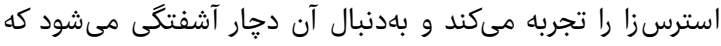

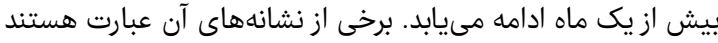

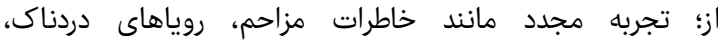

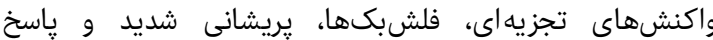

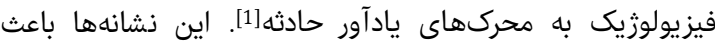
اختلال در عملكرد شغلى، اجتماعى و تحصيلى افراد مبتلادي به عادئ علايم 


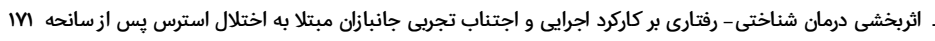

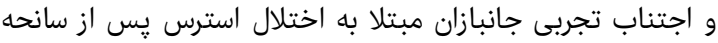

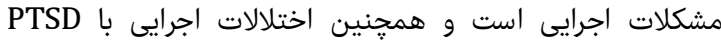

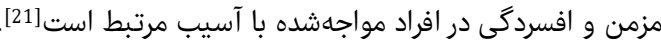

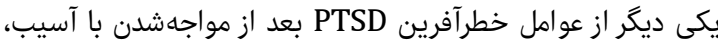

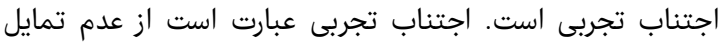

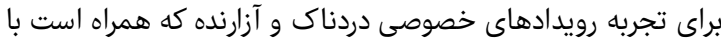

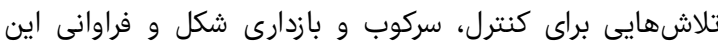

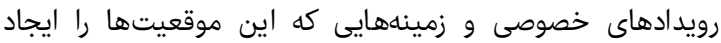

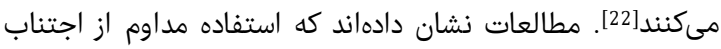

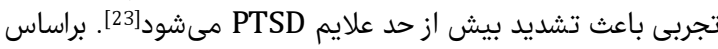

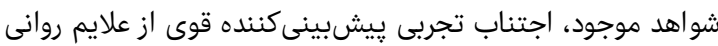

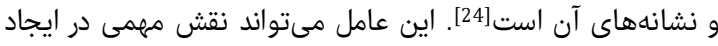

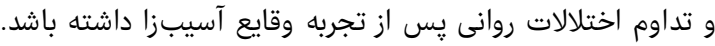

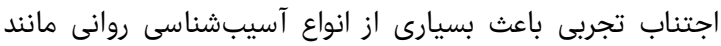

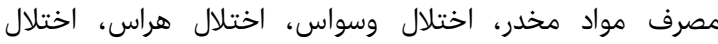

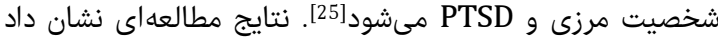

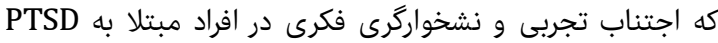

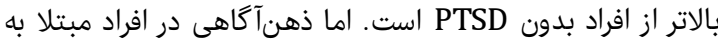

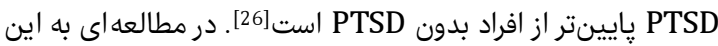

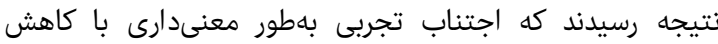

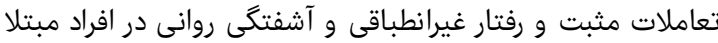

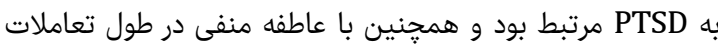

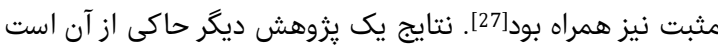

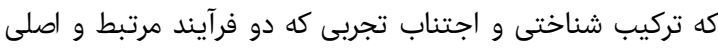

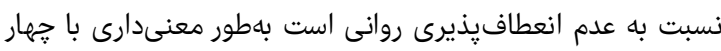

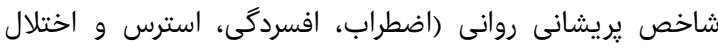

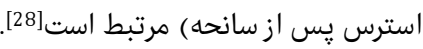

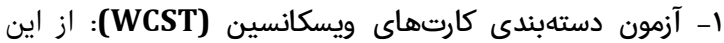

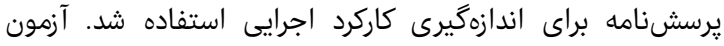

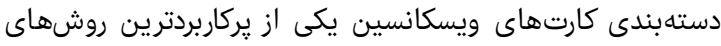

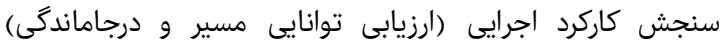

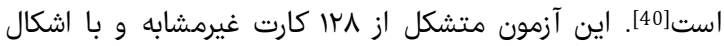

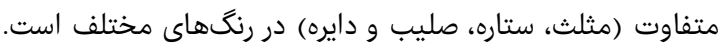

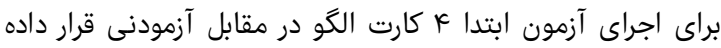

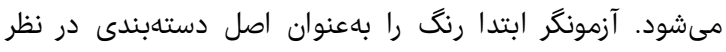

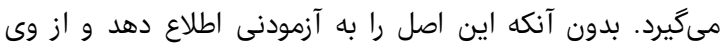

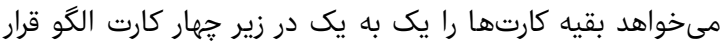

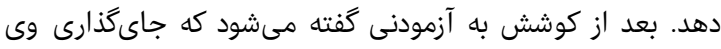

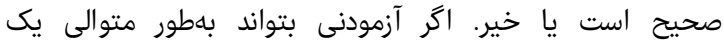

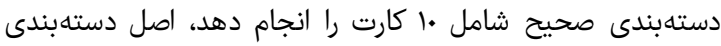

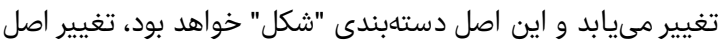

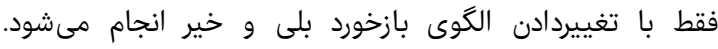

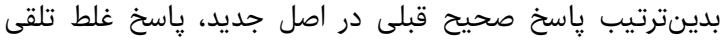

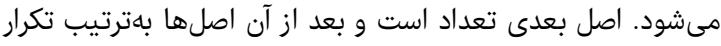

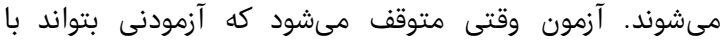

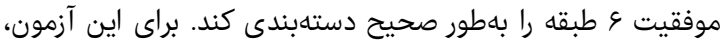

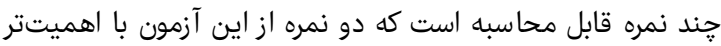

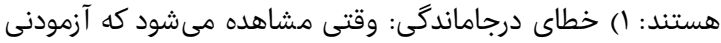

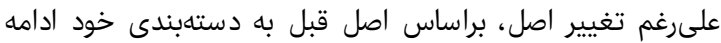

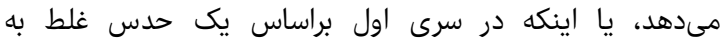

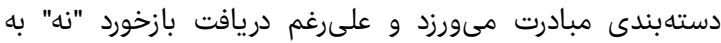

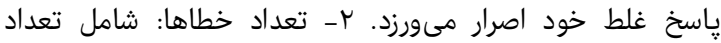
خطاهايى است كه يس از درنظركرفتن قاعده جديد و وردار دريافت 


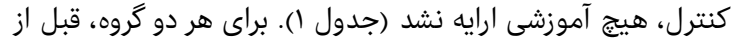

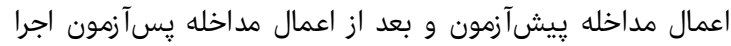

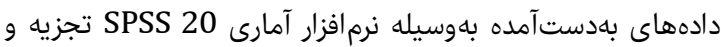

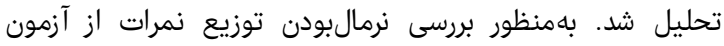

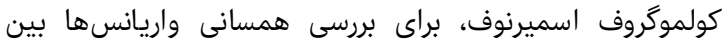

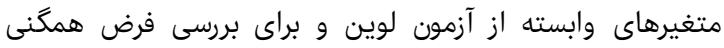

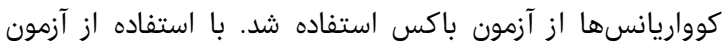

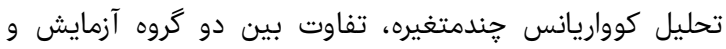

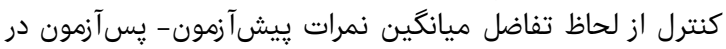

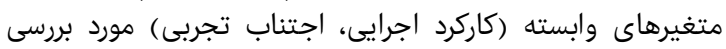

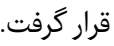

يافتهها

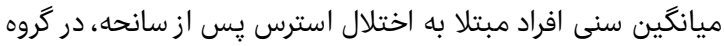

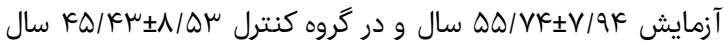

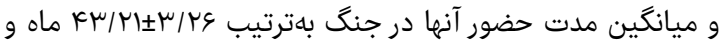
ميان

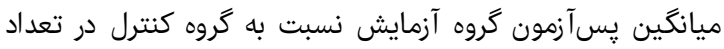

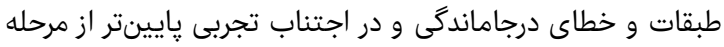

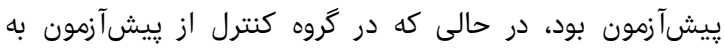

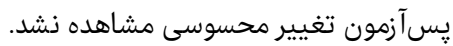

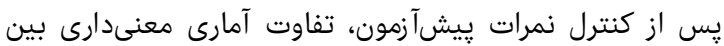

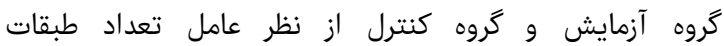

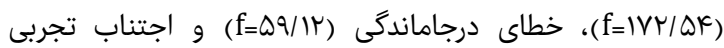

$$
\text { (f=|K/|ৎ) }
$$

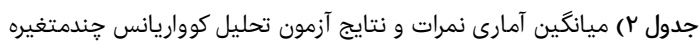

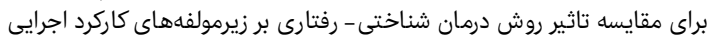

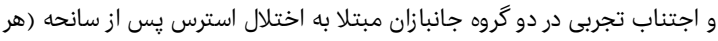

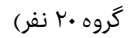

\begin{tabular}{|c|c|c|c|c|}
\hline ميزان تاثير & معنىدارى & كروه كنترل & كروه آزمايش & متغيرها \\
\hline.$/ 1 \Lambda$ &.$\% k^{k}$ & $q / \mu \xi_{ \pm} \mu / r \Delta$ & $|r / Q K \pm \Delta / Y|$ & تعد ادله يِقات آزمون \\
\hline$\cdot|r|$ & $.1 .9 V$ & $\Lambda / \Delta \xi \pm r / \cdot 1$ & $\mid r / \mu r \pm r / r k$ & خطاى درجاماندگى \\
\hline .110 &.$/ \Delta \Lambda$ & $\mu y / r \mu \pm r / \cdot \mu$ & $k \varepsilon / \varepsilon q \pm \mu / V q$ & اجتناب تجربى \\
\hline$\cdot|\Delta|$ & $.1 . .1$ & $q / / r \pm k / / k$ & 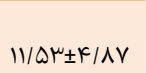 & تعد ادحله يسقات آزمون \\
\hline.$|8|$ &. $\mid .1$ & $\checkmark / r \vee \pm \mu / Q \varsigma$ & $9 / \wedge \kappa \pm F / .9$ & خطاى درجاماندگى \\
\hline . $/ \Delta \mu$ & $.1 . .1$ & $\mu \Delta / \Delta q \pm r / \cdot 1$ & $r \cdot / 1 \Delta \pm r / 1 V$ & اجتناب تجربى \\
\hline
\end{tabular}

يزوهش حاضر، با هدف بررسى درمان شناختى - رفتارى بر كاركرد

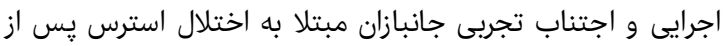

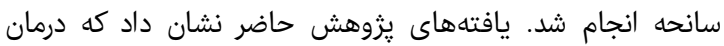

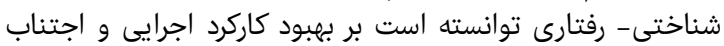

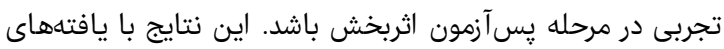

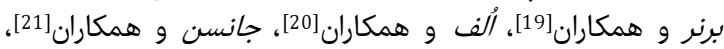

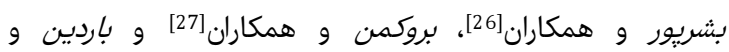

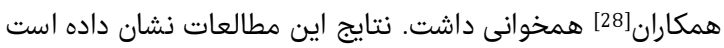

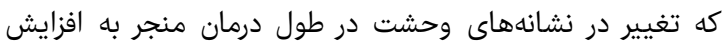

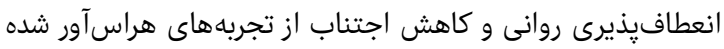

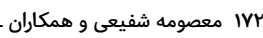

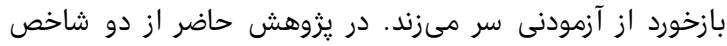

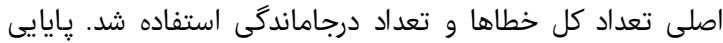

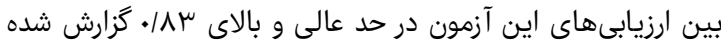

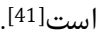

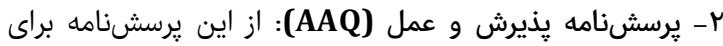

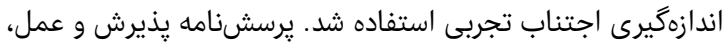

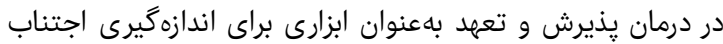

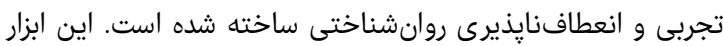

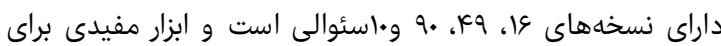

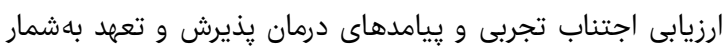

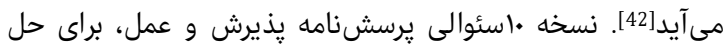

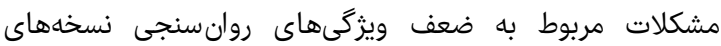

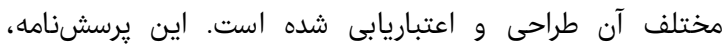

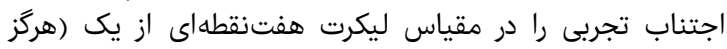

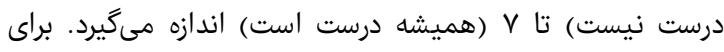

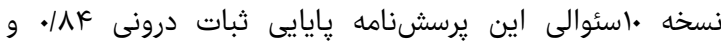

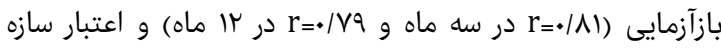

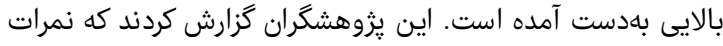

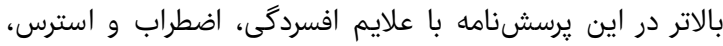

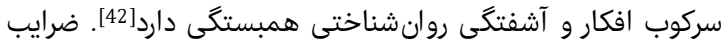

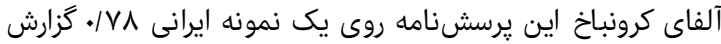

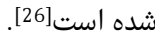

جدول () خلاصه فعاليتهاى انجامشده در جلسات كروه درمانى براى جانبازان مبتلا به اختلال استرس يس إز سانحه

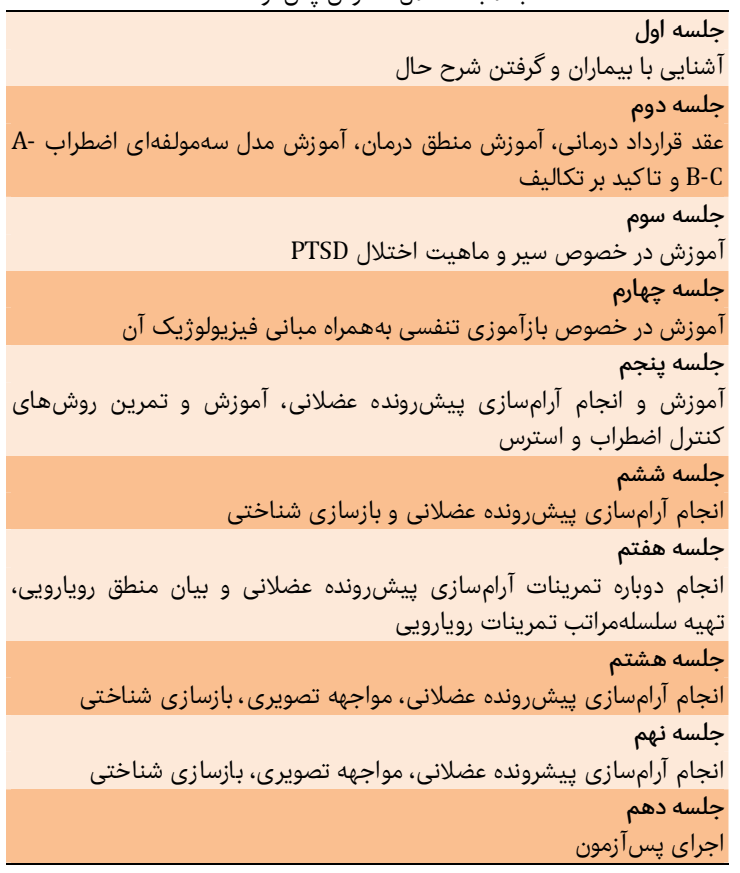

يس از اخذ رضايت كتبى و شفاهى از جانبازان مبنى بر عدم

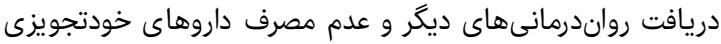

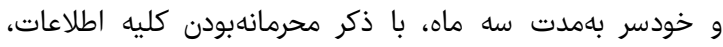

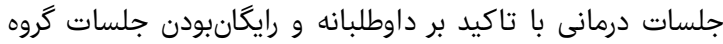

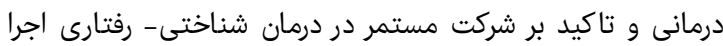

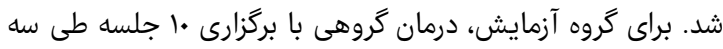

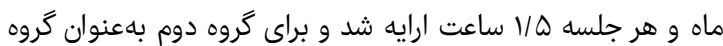




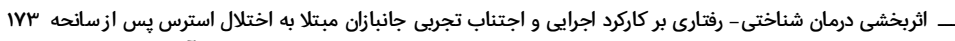

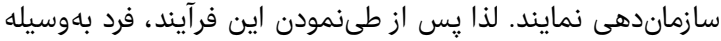

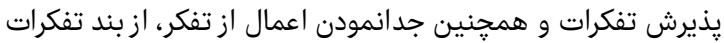

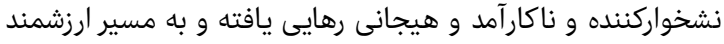

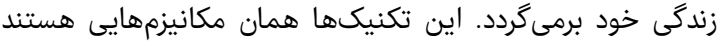

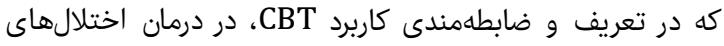

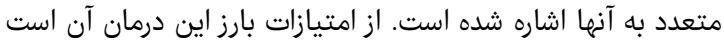

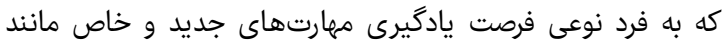

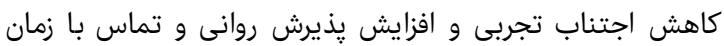

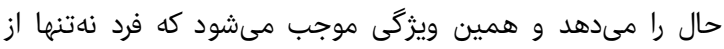

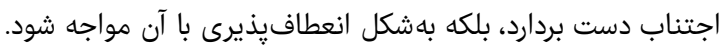

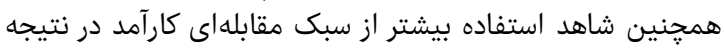

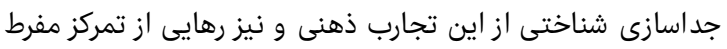

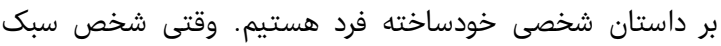

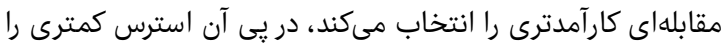

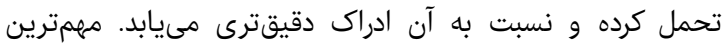

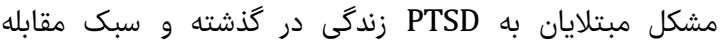

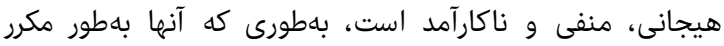

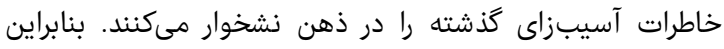

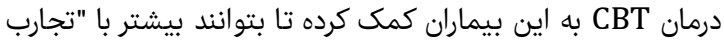
زندكى اينجا و كنونى" خود در تماس بـ باشند

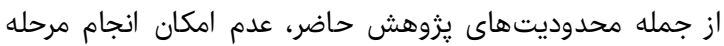

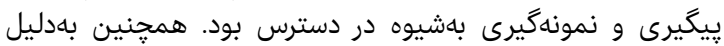

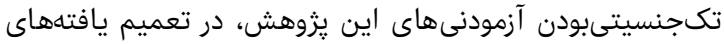

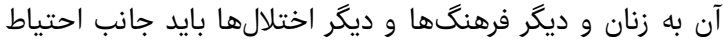
رعايت شود.

با توجه به اثربخشبودن درمان گروهى CBT بر علايم PTSD و

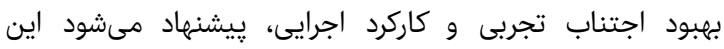

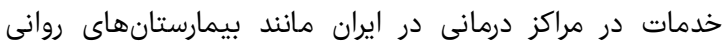

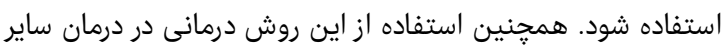

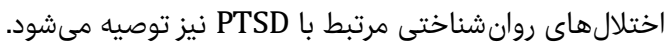

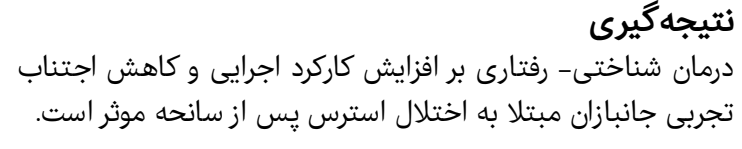

تشكر و قدر دانى: از همكارى و همراهى صميمانه كاركنان

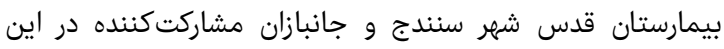

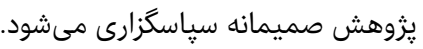

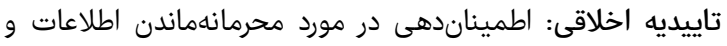

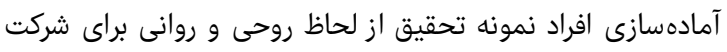

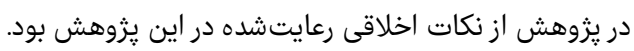

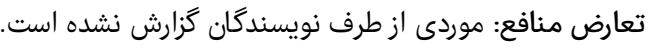

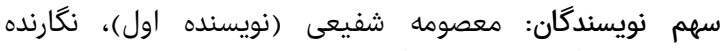

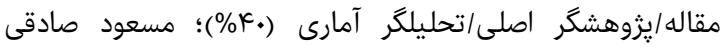

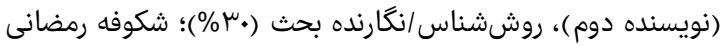

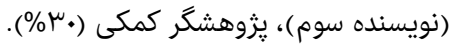

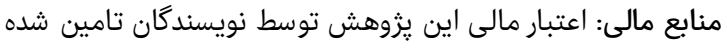
است.

\section{منابع}

1- American Psychiatric Association. Diagnostic and statistical manual of mental disorders (DSM). $5^{\text {th }}$ edition.

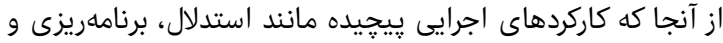

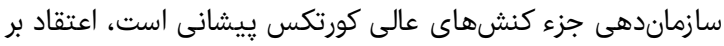

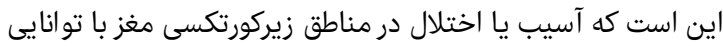

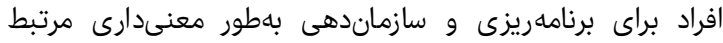

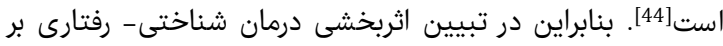

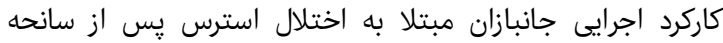

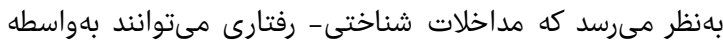

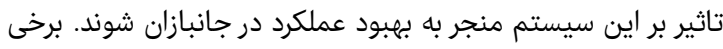

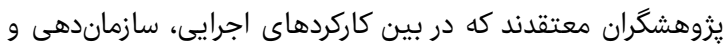

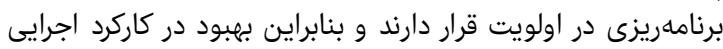

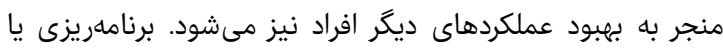

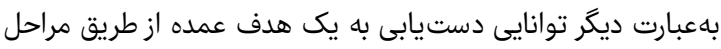

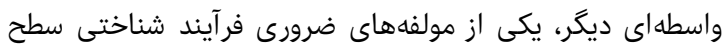

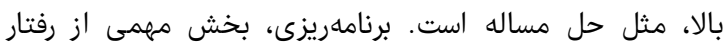

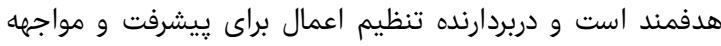

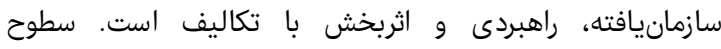

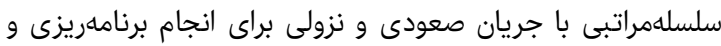

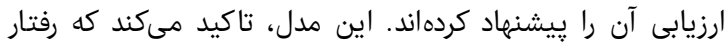

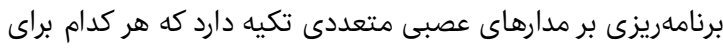

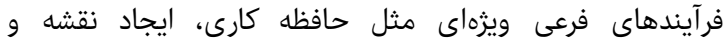

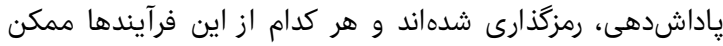

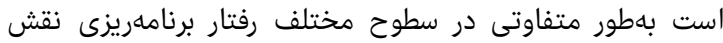

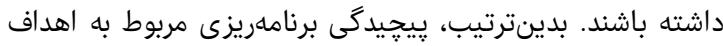

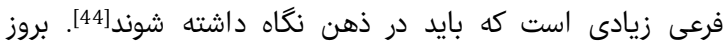

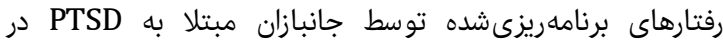

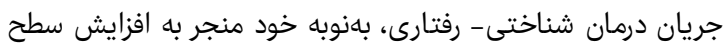

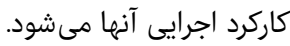

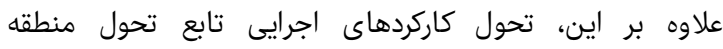

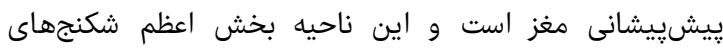

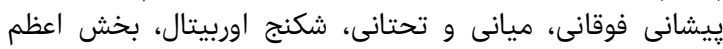

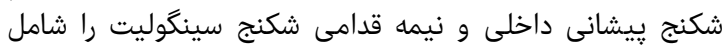

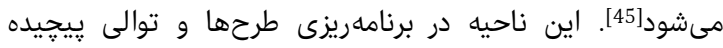

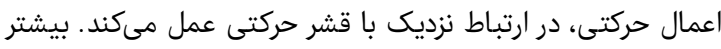

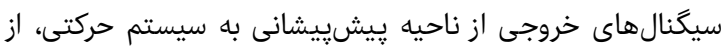

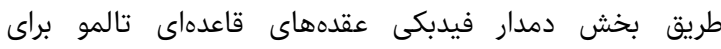

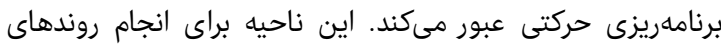

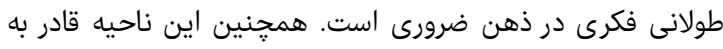

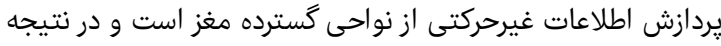

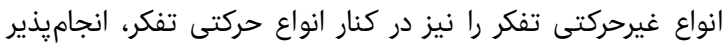

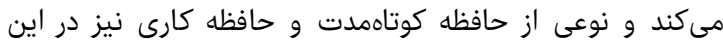

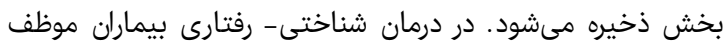

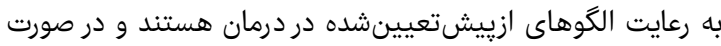

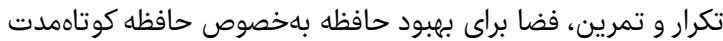

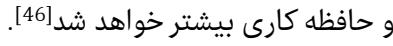

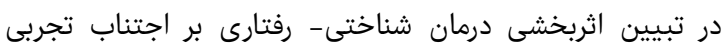

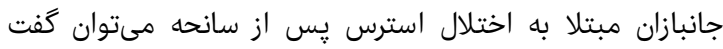

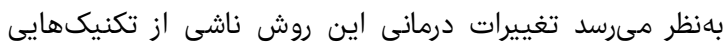

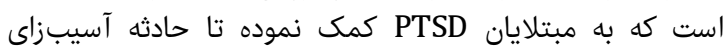

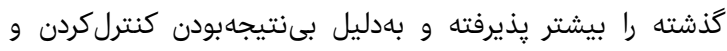

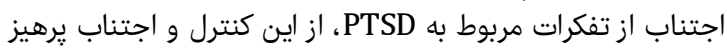

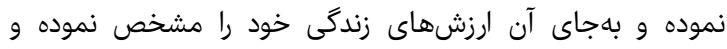

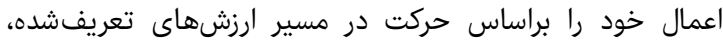


17- Devine H, Stewart SH, Watt MC. Relationship between anxiety sensitivity and dimensions of a alexithymia in a young adult sample. J Psychosom Res. 1999;47(2):145-58.

18- Gholamrezay S, Azizi A, Esmail M, Esmail S, Peyda N. Nature's role in predicting quality of positive cognitive emotion regulation strategies in pris-oners. Q J Soc Work. 2014;3(3):31-8. [Persian]

19- Brenner LA, Bahraini N, Homaifar BY, Monteith LL, Nagamoto H, Dorsey-Holliman B, et al. Executive Functioning and Suicidal behavior among veterans with and without a history of Traumatic Brain Injury. Arch Phys Med Rehabil. 2015;96(8):1411-8.

20- Olff M, Polak AR, Witteveen AB, Denys D. Executive function in posttraumatic stress disorder (PTSD) and the influence of comorbid depression. Neurobiol Learn Mem. 2014;112:114-21.

21- Johnsen GE, Kanagaratnam P, Nordling T, Wilberg C, Asbjørnsen AE. Psychophysiological responses to an executive task in refugees with chronic PTSD and comorbid depression. Nordic Psychology. 2017;69(3):177-94.

22- Hayes SC, Wilson KG, Gifford EV, Follette VM, Strosahl K. Experiental avoidance and behavioral disorders: A functional dimensional approach to diagnosis and treatment. J Consult Clin Psychol. 1996;64(6):1152-68.

23- Tull MT, Gratz KL, Salters K, Roemer L. The role of experiential avoidance in posttraumatic stress symptoms and symptoms of depression, anxiety, and somatization. J Nerv Ment Dis. 2004;192(11):754-61.

24- Marx BP, Sloan DM. Peritraumatic dissociation and experiential avoidance as predictors of posttraumatic stress symptomatology. Behav Res Ther. 2005;43(5):569-83.

25- Pickett SM, Bardeen JR, Orcutt HK. Experiential avoidance as a moderator of the relationship between behavioral inhibition system sensitivity and posttraumatic stress symptoms. J Anxiety Disord. 2011;25(8):1038-45.

26- Basharpoor S, Shafiei M, Daneshvar S. The comparison of experimental avoidance, [corrected] mindfulness and rumination in trauma-exposed individuals with and without posttraumatic stress disorder (PTSD) in an Iranian sample. Arch Psychiatr Nurs. 2015;29(5):279-83.

27- Brockman C, Snyder J, Gewirtz A, Gird SR, Quattlebaum J, Schmidt N, et al. Relationship of service members' deployment trauma, PTSD symptoms, and experiential avoidance to postdeployment family reengagement. J Fam Psychol. 2016;30(1):52-62.

28- Bardeen JR, Fergus TA. The interactive effect of cognitive fusion and experiential avoidance on anxiety, depression, stress and posttraumatic stress symptoms. J of Context Behav Science. 2016;5(1):1-6.

29- LaMontagne L, Hepworth JT, Cohen F, Salisbury MH. Cognitive-behavioral intervention effects on adolescents' anxiety and pain following spinal fusion surgery. Nurs Res.2003;52(3):183-90.

30- Beck AT. The current state of cognitive therapy: A 40-year retrospective. Arch Gen Psychiatry. 2005;62(9):953-9.

31- Roiser JP, Elliott R, Sahakian BJ. Cognitive mechanisms of treatment in depression. Neuropsychopharmacology. 2012;37:117-36.

32- Decker SE, Kiluk BD, Frankforter T, Babuscio T, Nich
Washington, DC London: American Psychiatric Association; 2013.

2- Chossegros L, Hours M, Charnay P, Bernard M, Fort E, Boisso D, et al. Predictive factors of chronic posttraumatic stress disorder 6 months after a road traffic accident. Accid Anal Prev. 2011;43(1):471-7.

3- Ehlers A, Clark DM, Hackmann A, McManus F, Fennell M. Cognitive therapy for post-traumatic stress disorder: Development and evaluation. Behav Res Ther. 2005;43(4):413-31.

4- ter Kuile MM, Weijenborg PM. A cognitive-behavioral group program for women with vulvar vestibulitis syndrome (VVS): Factors associated with treatment success. J Sex Marital Ther. 2006;32(3):199-213.

5- Roberts NP, Roberts PA, Jones N, Bisson JI. Psychological interventions for post-traumatic stress disorder and comorbid substance use disorder: A systematic review and meta-analysis. Clin Psychol Rev. 2015;38:25-38.

6- Moahghagh Motlagh J, Momtazy S, Mousavi Nasab SN, Arabs A, Sabory E, Sabory A. Investigating symptoms of post traumatic stress disorder in chemical warfare victims compared to non-chemical male veterans. J Mashhad Univ Med Sic. 2013;56(6):362-8. [Persian]

7- Horesh D, Lowe SR, Galea S, Aiello AE, Uddin M, Koenen K. An in depth look into PTSD depression comorbidity: A longitudinal study of chronically-exposed Detroit residents. J Affect Disord. 2017;208:653-61.

8- Tay AK, Rees S, Chan J, Kareth M, Silove D. Examining the broader psychosocial effects of mass conflict on PTSD symptoms and functional impairment amongst West Papuan refugees resettled in Papua New Guinea (PNG). Soc Sci Med. 2015;132:70-8.

9- McDevitt-Murphy ME, Luciano MT, Tripp JC, Eddinger JE. Drinking motives and PTSD-related alcohol expectancies among combat veterans. Addict Behav. 2017;64:217-22.

10- Bowen S, De Boer D, Bergman AL. The role of mindfulness as approach-based coping in the PTSDsubstance abuse cycle. Addict Behav. 2017;64:212-6.

11- Simons JS, Simons RM, O'Brien C, Stoltenberg SF, Keith JA, Hudson JA. PTSD, alcohol dependence, and conduct problems: Distinct pathways via lability and disinhibition. Addict Behav. 2017;64:185-93.

12- Brown WJ, Wilkerson AK, Milanak ME, Tuerk PW, Uhde TW, Cortese BM, et al. An examination of sleep quality in veterans with a dual diagnosis of PTSD and severe mental illness. Psychiatry Res. 2017;247:15-20.

13- Poindexter EK, Mitchell SM, Jahn DR, Smith PN, Hirsch JK, Cukrowicz KC. PTSD symptoms and suicide ideation: Testing the conditional indirect effects of thwarted interpersonal needs and using substances to cope. Personal Individ Differ. 2015;77:167-72.

14- Rick AD, Vanheules S. Alexithymia and DSM-IV personality disorder traits in alcoholic inpatients: A study of the relation between both constructs. Personal Individ Differ. 2007;43(1):119-29.

15- Haviland MG, Hendryx MS, Shaw DG, Henry JP. Alexithmia in women and men hospitalized for psychoactive substance dependence. Compr Psychiatry. 1994;35(2):124-8.

16- Rufer M, Hand I, Braatz A, Alsleben H, Fricke S, Peter $\mathrm{H}$. Aprospective study of alexithymia in obsessivecompulsive patients treated with multimodal cognitivebehavioral therapy. Psychother Psychosom. 2004;73(2):101-6. 


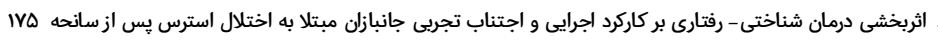

Pistorello J, Toarmino D, et al. Measuring experiential avoidance: A preliminary test of a working model. Psychol rec. 2004;54(4):553-78.

40- Bedard MJ, Joyal CC, Godbout L, Chantal S. Executive functions and the obsessive-compulsive disorder: On the importance of subclinical symptoms and other concomitant factors. Arch Clin Neuropsychol. 2009;24(6):585-98.

41- Martínez-Arán A, Vieta E, Reinares M, Colom F, Torrent C, Sánchez-Moreno J, et al. Cognitive function across manic or hypomanic, depressed, and euthymic states in bipolar disorder. Am J Psychiatry. 2004;162(2):262-70.

42- Bond FW, Hayes SC, Baer RA, Carpenter KM, Guenole $\mathrm{N}$, Orcutt $\mathrm{HK}$, et al. Preliminary psychometric properties of the Acceptance and Action Questionnaire-II: A revised measure of psychological inflexibility and experiential avoidance. Behav Ther. 2011;42(4):676-88.

43- Gloster AT, Klotsche J, Gerlach AL, Hamm A, Strohle A, Gauggel S, et al. Timing matters: change depends on the stage of treatment in cognitive behavioral therapy for panic disorder with agoraphobia. J Consult Clin Psychol, 2014;82(1):141-53.

44- Ardila A. On the evolutionary origins of executive functions. Brain Cogn. 2008;68(1):92-9.

45- Clawson M. Play: Essential for all children. A position paper of the association for childhood education international childh educat play: Essential for all children. 2002;79(1):116-93.

46- Hanney L, Kozlowska K. Healing traumatized children: Creating illustrated storybooks in family therapy. Fam Process. 2002;41(1):37-65.
C, Carroll KM. Just showing up is not enough: Homework adherence and outcome in cognitive-behavioral therapy for cocaine dependence. J Consult Clin Psychol. 2016;84(10):907-12.

33- Johnsen TJ, Friborg 0 . The effects of cognitive behavioral therapy as an anti-depressive treatment is falling: A meta-analysis. Psychol Bull. 2015;141(4):74768.

34- Koenig HG, Pearce MJ, Nelson B, Shaw SF, Robins CJ, Daher NS, et al. Religious vs. Conventional Cognitive Behavioral Therapy for Major Depression in Persons With Chronic Medical Illness: A Pilot Randomized Trial. J Nerv Ment Dis. 2015;203(4):243-51.

35- Davies CD, Niles AN, Pittig A, Arch JJ, Craske MG. Physiological and behavioral indices of emotion dysregulation as predictors of outcome from cognitive behavioral therapy and acceptance and commitment therapy for anxiety. J Behav Ther Exp Psychiatry. 2015;46:35-43.

36- Ost LG, Riise EN, Wergeland GJ, Hansen B, Kvale G. Cognitive behavioral and pharmacological treatments of OCD in children: A systematic review and meta-analysis. J Anxiety Disord. 2016;43:58-69.

37- Gregory B, Peters L. Changes in the self during cognitive behavioural therapy for social anxiety disorder: A systematic review. Clin Psychol Rev.2017;52:1-18.

38- Miyahira SD, Folen RA, Hoffman HG, Garcia-Palacios A, Schaper KM. Effectiveness of brief VR treatment for PTSD in war-fighters: A case study. Stud Health Technol Inform. 2010;154:214-9.

39- Hayes SC, Strosahl K, Wilson KG, Bissett RC, 